\title{
Density Waves in the Flows of Granular Media
}

\author{
Jysoo Lee \\ HLRZ-KFA Jülich, Postfach 1913, W-5170 Jülich, Germany
}

(July 29, 2018)

\begin{abstract}
We study density waves in the flows of granular particles through vertical tubes and hoppers using both analytic methods and molecular dynamics (MD) simulations. We construct equations of motion for quasi one-dimensional systems. The equations, combined with the Bagnold's law for friction, are used to describe the time evolutions of the density and the velocity fields for narrow tubes and hoppers. The solutions of the equations can have two types of density waves, kinetic and dynamic. For tubes, we can show the existence of kinetic waves, and obtain the condition for dynamic waves for tubes from the equations. For hoppers, we obtain the solutions of the equations up to the first order of the opening angle, which also show the existence of kinetic waves. We reproduce density waves in the MD simulations for tubes. The waves are believed to be kinetic based on a few evidences, including a well defined flux-density curve. In MD simulations of flows in hoppers, we find density waves, which are also believed to be kinetic.

$05.40+\mathrm{j}, 46.10+\mathrm{z}, 62.20-\mathrm{x}$
\end{abstract}

Typeset using REVTEX 


\section{INTRODUCTION}

Systems of granular particles (e.g. sands) exhibit many interesting phenomena [1] [3]. The formations of a spontaneous heap [4 4 and convection cells 8 [12 under vibration, and the segregation of particles [13 [18] are just a few examples. These phenomena are consequences of unusual, and often complex, dynamical responses of the system. Considering the complexity of the dynamics, one is tempted to first have comprehensive understanding of the dynamics of granular media in relatively simple geometries, and proceed to more complicated situations. Even for the simple geometries like shear cells, vertical tubes and hoppers, granular media still show complex dynamics. For example, granular particles in a shear cell show not only non-Newtonian behaviors [19 22], but also stick-slip motions [23] as well as density waves [24,25]. Here, we study the flows of granular particles through vertical tubes and hoppers, whose geometries are as simple as shear cells, but are less well studied. In granular flows through vertical tubes, Pöschel [26] found that the particles do not flow uniformly, but form regions of high density which travel with velocity different from that of the center of mass. The mechanism for the traveling density pattern or the density wave is, however, not clearly understood. The density waves are also found in outflows through hoppers [27,28]. Especially, Baxter et al [28] show that the velocities of the density waves are dependent on the opening angle of hoppers, and even their directions can be changed. Furthermore, they find the density waves only when they mix some amount of rough sands with smooth sands. In MD simulations of hopper, the density field is found out to be non-uniform [29]. Again, the mechanism for the density wave is not clearly understood.

We first construct equations of motion for quasi one-dimensional systems. With the Bagnold's law for friction [19], the equations are used to describe the time evolutions of the density and the velocity fields for narrow tubes and hoppers. The solutions of the equations can have two types of density waves, kinetic and dynamic. The dynamic waves are similar to sound waves, while kinetic waves are due to the kinematics of the equations [30]. For tubes, we can show the existence of kinetic waves, and obtain the condition for dynamic 
waves by a linear stability analysis. We also study the effects of static friction, and find that it changes the qualitative behaviors of the flows. For hoppers, we obtain the solutions of the equations up to the first order of the opening angle, and also show the existence of kinetic waves. Next, we study the systems using molecular dynamics (MD) simulations. For tubes, we reproduce density waves, and we find a few strong evidences that the waves are of kinetic nature, which includes a well defined flux-density curve and the density dependence of the velocities of the waves. We also study the existence of dynamic waves by changing the inelasticity of the particles, and do not find dynamics waves. In MD simulations of flows in hoppers, we find density waves, which are believed to be kinetic waves due to the strong correlation between the density and the velocity fields. For hoppers with periodic boundary conditions, we find additional density waves, which eventually dominates. We find a few evidences that the additional waves are of dynamic nature. We, however, do not find dynamic waves in hopper with open boundary conditions. The results obtained by these theoretical and numerical studies are compared with the experiments.

This paper is organized as follows. In Sec. ㅍ. we present theoretical results. Detailed discussions on the definitions and properties of dynamic and kinetic waves are given in Sec. ПА. The kinetic and dynamic waves in tubes are discussed in Sec. ПB 1 and Sec. ПB2, respectively. Also, kinetic waves in hoppers are discussed in Sec. IIC. We present the numerical results in Sec. III. First, we define the interactions between the particles in Sec. IIIA. The results for kinetic and dynamic waves in tubes are given in Sec. IIIB, and the results for hoppers are discussed in Sec. [II . Brief summary as well as the limitations of this work are given in Sec. IV].

\section{THEORETICAL APPROACH}




\section{A. Dynamic and kinetic waves}

Here, we construct equations of motion for flows in one-dimension, which will later be used to describe the time evolutions of fields in vertical tubes and hoppers. Let us define $\rho(x, t)$ and $v(x, t)$ be the density and the velocity at position $x$ and time $t$, respectively. If we require the mass of the system to be conserved,

$$
\frac{\partial}{\partial t} \rho+\frac{\partial}{\partial x}(\rho v)=0
$$

Other equation comes from the momentum conservation,

$$
\rho \frac{\partial}{\partial t} v+\rho v \frac{\partial}{\partial x} v=F(x, t)
$$

where $F(x, t) d x$ is the total force acting on the mass within $[x, x+d x]$ at time $t$.

There are two distinct mechanisms by which density patterns can travel. One is due to the instability of uniform-density flows to density fluctuations ("dynamic wave"), and the other is of kinetic origin ("kinetic wave") [30]. We now discuss both types of waves in detail, paying particular attention to their differences. In order to make the discussion more concrete, we use a specific form of the force

$$
F(x, t)=\rho-\frac{\partial}{\partial x} \rho-\rho^{\nu} v^{\mu}
$$

where $\mu$ and $\nu$ are constants. The form of Eq. (2), motivated from a granular flow in a tube, is chosen such that the discussion becomes more clear. The followings are, however, applicable for any form of the force.

We first consider the dynamic wave. The equations of motion Eqs. (1) with the force given by Eq. (2) have a solution of uniform density $\rho_{o}$,

$$
\begin{aligned}
& \rho(x, t)=\rho_{o}, \\
& v(x, t)=\rho_{o}^{(1-\nu) / \mu} .
\end{aligned}
$$

We study the stability of the solution Eq. (3) by a linear stability analysis. For simplicity, we consider the case of $\rho_{o}=1$. The trial solution for the time evolution of a perturbation of Eq. (3) is 


$$
\begin{aligned}
& \rho(x, t)=1+\epsilon_{\rho} \exp [i(k x-\omega t)] \\
& v(x, t)=1+\epsilon_{\mathrm{v}} \exp [i(k x-\omega t)],
\end{aligned}
$$

where $\epsilon_{\rho}$ and $\epsilon_{\mathrm{v}}$ are constants. We want to study the time evolution in a linear approximation, where we consider terms up to the first order of $\epsilon$. The analysis is valid only if a perturbation term is much smaller than unity. Substituting Eq. (幽 into Eqs. (1), and discard terms higher order than $\epsilon$, we obtain

$$
\begin{aligned}
(\omega-k) \epsilon_{\rho}-k \epsilon_{\mathrm{v}} & =0 \\
{[k+i(1-\nu)] \epsilon_{\rho}+[(k-\omega)-i \mu] \epsilon_{\mathrm{v}} } & =0,
\end{aligned}
$$

whose solution is given by

$$
\omega=\frac{2 k-i \mu \pm \sqrt{4 k[k+i(1-\nu)]-\mu^{2}}}{2} .
$$

The density fluctuation travels with velocity $\operatorname{Re}(\omega) / k$, where $\operatorname{Re}(\omega)$ is the real part of $\omega$. If $\operatorname{Im}(\omega)$ (the imaginary part of $\omega$ ) is positive, the perturbation grows with time, makes uniform-density flow unstable. This mechanism for creating density waves, which is based on the instability of the equations, is called dynamic waves.

We now discuss the mechanism for kinetic waves. For an excellent introduction to the subject, please see Ref. [30]. Consider a case that the system is divided into two uniformdensity flow regions with densities $\rho_{a}$ and $\rho_{b}$, and the velocities of each region is determined by Eq. (3) (Fig. 1). The equations of motion Eqs. (11) imply that density and velocity field $(\rho(x, t)$ and $v(x, t))$ inside each domains do not change. The system evolve only by moving the interface. Let $U$ be the velocity of the interface. We also set the initial position of the interface to be 0 without losing generality. Then, the position of the interface at time $t$ is $U t$. At a given time, we choose an interval $[-\epsilon, \epsilon]$ which includes the interface. We then integrate Eq. (1a) in the interval

$$
\begin{aligned}
\int_{-\epsilon}^{\epsilon} \frac{\partial}{\partial t} \rho+\frac{\partial}{\partial x}(\rho v) & =\frac{\partial}{\partial t}\left[(\epsilon+U t) \rho_{a}+(\epsilon-U t) \rho_{b}\right]+\left.\rho v\right|_{-\epsilon} ^{\epsilon} \\
& =\left(\rho a-\rho_{b}\right) U+j_{b}-j_{a}=0,
\end{aligned}
$$


where the flux $j$ is defined to be $\rho v$. Therefore, the interface, or any density fluctuations, travels with velocity

$$
U=\frac{j_{a}-j_{b}}{\rho_{a}-\rho_{b}}=\frac{\Delta j}{\Delta \rho} .
$$

Since the above mechanism for the travel of density fluctuations is a consequence of a kinetic equation (mass conservation, Eq. (1a)), it is called kinetic wave. Unlike dynamics waves, the fluctuations in kinetic waves can not be amplified, the system can only reorganize existing fluctuations. The fluctuations are created by initial conditions or by noises in the dynamics. Furthermore, kinetic waves, in most cases, decay into uniform flows [30].

In general, flows in 1-d have both kinetic and dynamic waves. If a system is stable under density fluctuations, existing dynamic waves will decay exponentially, and only kinetic waves can survive. On the other hand, if the system is unstable, the amplitudes of the dynamic waves will grow, and they eventually dominate over kinetic waves. In order to illustrate this point, we integrate numerically Eqs. (11) to get $\rho(x, t)$ and $v(x, t)$, where the force is given by Eq. (2). The integration is done using the staggered leapfrog method [31] with the time step 0.001. We also use a periodic boundary condition. The initial conditions are that $\rho(x, 0)$ is a short pulse of higher density $(1 \%)$ on the top of background density of unity, and $v(x, 0)$ is given by Eq. (3). We first study the case of $\mu=2, \nu=-1$. The behavior of the dynamic waves is studied by a linear stability analysis. Equation (6) gives the dependence of $\omega$ on $k$ to be $2 k,-2 i$. There is one marginally stable mode of velocity 2 , and one stable mode of velocity 0 . On the other hand, the flux is given as

$$
j \equiv \rho v=\rho^{(\mu-\nu+1) / \mu},
$$

and the velocity of kinetic wave, determined by Eq. (8), is approximately 2. Since the system is not unstable to density fluctuations, the kinetic waves, not greatly affected by the dynamic waves, will survive for a long time (Fig. 2(a)). We now study the case of $\mu=-2, \nu=-1$. Following the same analysis, $\omega(k)$ is determined to be $2 k+2 i$ and 0 . One unstable mode of velocity 2 and one marginally stable mode of velocity 0 . The velocity of the kinetic wave 
is approximately 0 . The dynamic wave of increasing amplitude (that of the velocity 2) is dominating over the kinetic wave (Fig. 2(b)).

\section{B. Density waves in vertical tubes}

\section{Kinetic waves in vertical tubes}

We consider vertical tubes of narrow width, which are filled with granular material. The particles will flow down due to gravity. This motion will induce the forces between the particles as well as the friction forces by the inner walls of the tubes. We assume that these forces follow the Bagnold's law [19]. The law, first found in a rapid granular flow, implies that the components of the stress tensor $\tau_{i j}$ are proportional to the square of the shear rate $\dot{\gamma}$

$$
\tau_{i j}=\rho_{B} D^{2} f_{i j}(p) \dot{\gamma}^{2}
$$

Here, $\rho_{B}$ is the density of the material which forms the particles, and $p$ is the volume fraction of the particles. The density $\rho$ is, therefore, $\rho_{B}$ times $p$. Also, $D$ is the average

diameter of the particles, $f_{i j}(p)$ is a system dependent function. Bagnold predicted the shear rate dependence in rapid flows using a simple argument, which later confirmed by more elaborate calculations as well as experiments [2, 3, 20]. In this paper, we will use the law except the case that the velocities of the particles are considered to be very small.

Let $x$-axis ( $y$-axis) be the direction parallel (perpendicular) to the tube, where the positive $x$-direction is chosen to be upward. We consider the forces acting on the granular material contained in $[x, x+d x]$. Since we study only narrow tubes, we assume the material is homogeneous along the $y$ direction. There is a gravitational force given by $-\rho g W d x$, where $g$ is the gravitational acceleration, and $W$ the width of the tube. There also is a friction by the wall, which is $-\tau_{x y} d x$, and pressure by other particles $-d \tau_{x x} / d x D d x$. The total force $F(x, t)$ becomes 


$$
\begin{aligned}
F(x, t) & =-\rho_{B} p g W-\operatorname{sign}(v) \rho_{B} D^{2} f_{x y}(p)\left(\frac{v}{D}\right)^{2}-D \frac{\partial}{\partial x}\left[\rho_{B} D^{2} f_{x x}(p)\left(\frac{v}{D}\right)^{2}\right] \\
& =-\rho_{B} p g W-\operatorname{sign}(v) \rho_{B} f_{x y}(p) v^{2}-D \frac{\partial}{\partial x}\left[\rho_{B} f_{x x}(p) v^{2}\right]
\end{aligned}
$$

where $\operatorname{sign}(v)$ is the $\operatorname{sign}$ of $v$, and we assume the width of shear layer to be of order of $D$.

Having found the force, we now proceed to study kinetic waves in the system. We first consider the case of no static friction. The steady-state velocity $v_{s}(p)$ of a uniform flow is obtained from the condition that the total force acting on the granular material is zero. Since we consider uniform density flows, the force free condition gives

$$
v_{s}(p)=-\sqrt{p g W / f_{x y}(p)} .
$$

The flux $j(p)$ is given by $\rho_{B} p v_{s}(p)$, then the velocity of a small density fluctuation $U(p)$ becomes

$$
U(p)=\frac{\Delta j}{\Delta \rho} \simeq \frac{d j(p)}{d \rho}=\frac{1}{2} v_{s}(p) \frac{3 f_{x y}(p)-p d f_{x y}(p) / d p}{f_{x y}(p)}
$$

Therefore, the form of the force Eq. (11) allows density fluctuations to travel with velocity $U(p)$. The condition for the existence of kinetic waves is the balance between the forces, and the waves are not very sensitively dependent on the exact form of the forces. Therefore, they are very likely to be seen in the experiments.

We now consider the effects of static friction on the kinetic waves. By static friction, we mean one has to apply a finite force in order to break contacts between surfaces. There are two contributions to the normal stress on the wall. One is a static pressure $P_{s}$, which is independent of the motion of the particles. The other is due to the collisions of the particles on the wall $\tau_{y y}$, given by Eq. (10). The total friction force is the minimum of $\rho g W-\tau_{x y}$ and $\mu\left(\tau_{y y}+P_{s}\right)$, where $\mu$ is the friction coefficient. If the shear force (gravity and friction) is smaller than $\mu$ times the normal force, the total force on the particles is zero, and the particles form a stagnant zone of no movement [32].

Depending on the parameters, there can be two behaviors of the system. If the gravitational force $\rho g W$ is less than the friction due to static pressure $\mu P_{s}$, the particles can not 
move, and the system remains as a static column. If $\rho g W>\mu P_{s}$, the particles will start move. The steady-state velocity $v_{s}^{\prime}(p)$ for given packing fraction $p$ is,

$$
v_{s}^{\prime}(p)=-\sqrt{\frac{p g W-\mu P_{s} / \rho_{B}}{f_{x y}(p)}},
$$

which is very similar to Eq. (12). However, we have additional constraint that the shear force should exceed $\mu$ times the normal force, which gives

$$
\rho g W-\rho_{B} f_{x y}(p) v^{2}>\mu P_{s}+\mu \rho_{B} f_{y y}(p) v^{2}
$$

If we define $v_{c}(p)$ be the velocity that above equation becomes equality,

$$
v_{c}(p)=v_{s}^{\prime}(p) \frac{1}{1+\mu f_{y y}(p) / f_{x y}(p)}<v_{s}^{\prime}(p) .
$$

Since the inequality Eq. (15) is not satisfied for $v(p)>v_{c}(p)$, the particles can not reach their steady state. Instead, they forms a stagnant zone. If we assume that the particles are stopped completely in the stagnant zone, the distances between the zones $\mathcal{D}(p)$ are constant, which is the distance needed for the particles to be accelerated to $v_{c}(p)$ from the stopped state. We can calculate the distance $\mathcal{D}(p)$ as follows. The velocity of the particles, obtained by integrating the Newton's equation with the friction force of Eq. (15), becomes

$$
v(t, p)=v_{s}^{\prime}(p) \tanh \left[v_{s}^{\prime}(p)\left(g-\frac{\mu P_{s}}{\rho W}\right) t\right]
$$

The distance covered by the particles during the interval $\left[0, t_{c}\right]$ becomes $\mathcal{D}(p)$, where $t_{c}$ is given by $v\left(t_{c}, p\right)=v_{c}(p)$. Integrating Eq. (17), we obtain

$$
D(p)=-\frac{v_{s}^{\prime 2}(p)}{2\left(g-\mu P_{s} / \rho W\right)} \ln \left(1-\gamma^{2}\right),
$$

where $\gamma=1 /\left(1+\mu f_{y y}(p) / f_{x y}(p)\right)$. The distance $\mathcal{D}(p)$ increases as $\mu$ is decreased, and it diverges when $\mu \rightarrow 0$.

We now summerize the results obtained in this section. If static friction is not present, the particles in vertical tubes form density waves travels with velocity $U(p)$. When we take into account static friction, the system forms periodic stagnant zones with their separations $\mathcal{D}(p)$. 


\section{Dynamic waves in vertical tubes}

In this section, we study the stability of the uniform-density flows through vertical tubes under density and velocity fluctuations. We will follow the analysis presented in Sec. ПIA. We start with the equations of motion Eqs. (11) with the force given by Eq. (11). Here, we do not consider static friction. The uniform-density flow solution with the density $\rho_{o}$ is

$$
\begin{aligned}
& \rho(x, t)=\rho_{o} \equiv \rho_{B} p_{o} \\
& v(x, t)=v_{o} \equiv-\sqrt{\frac{p_{o} g W}{f_{x y}\left(p_{o}\right)}} .
\end{aligned}
$$

We study the stability of the solution by studying the time evolution of the perturbation from the uniform-density flow, given by

$$
\begin{aligned}
& p(x, t)=p_{o}+\epsilon_{p} \exp [i(k x-\omega t)] \\
& v(x, t)=v_{o}+\epsilon_{v} \exp [i(k x-\omega t)]
\end{aligned}
$$

Substituting Eqs. (20) into Eq. (1), and consider only up to the first terms of $\epsilon$, we obtain

$$
\begin{array}{r}
\left(k v_{o}-\omega\right) \epsilon_{p}+p_{o} k \epsilon_{v}=0 \\
{\left[g-\frac{v_{o}^{2}}{W}\left(\frac{d f_{x x}}{d p}-i k D \frac{d f_{x y}}{d p}\right)\right] \epsilon_{p}+\left[i\left(k v_{o}-\omega\right)-\frac{2 v_{o}}{W}\left(f_{x y}-i k D f_{x x}\right)\right] \epsilon_{v}=0 .}
\end{array}
$$

Since the two equations in Eq. (21) are valid for any values of $\epsilon_{p}$ and $\epsilon_{v}$, the solution is

$$
\Omega^{2}-i \frac{2 v_{o}}{p_{o} W}\left(f_{x y}-i k D f_{x x}\right) \Omega+i k\left[g-\frac{v_{o}^{2}}{W}\left(\frac{d f_{x y}}{d p}-i k D \frac{d f_{x x}}{d p}\right)\right]=0,
$$

where $\Omega \equiv \omega-k v_{o}$. The stability of the uniform solution is determined by the imaginary part of $\omega$. Unfortunately, since $\omega$ depends on the exact form of the unknow function $f_{i j}$, we can not determine the stability of the flow. Similar stability analysis on shear cells [25] and dissipative gases [33] shows that the system is unstable for small coefficient of restitution $e$. Especially, in [33, this instability is traced back to the fact that pressure can decrease as the density is increased. Here, we can show that Eq. (22) has an unstable mode if $d f_{x x} / d p$ is sufficiently negative (smaller pressure for larger density), where the exact criterion is a complicated function of $k, f_{x y}$ and $f_{x x}$. The stability of the flow is later checked by MD 
simulations, where we will study the stability for various degrees of the inelasticity of the particles.

\section{Density waves in hoppers}

Consider a hopper with the opening angle $2 \theta$, where the width at position $x$ is given by $W(x)=W_{o}+2 \tan \theta x$ (Fig. 3(a)). Here, we consider only the positive ranges of $x$. The equations of motion for flows in hoppers are slightly different from those for tubes. Since the width $W$ is dependent on $x$, the conservation of mass implies

$$
W \frac{\partial}{\partial t} \rho+\frac{\partial}{\partial x}(W \rho v)=0
$$

instead of Eq. (1a). Equation (1b), which is a consequence of momentum conservation, still holds for hoppers, with a modified form of friction force. Since the sidewalls are tilted, the friction per unit length along the walls have two contributions. One is the component of $\tau_{x y}$ (friction force for tubes) parallel to the walls $\tau_{x y} \cos \theta$. Forces on the walls by the internal pressure $\tau_{x x}$ give additional contribution $\tau_{x x} \sin \theta \cos \theta$, where $\sin \theta$ is due to the cross-section for the collisions of particles to the walls. (Fig. 3(b)). Therefore, the total force becomes,

$$
F(x, t)=-\rho g+\rho_{B} v^{2}\left(f_{x y} \cos \theta+f_{x x} \sin \theta \cos \theta\right)-\rho_{B} D \frac{\partial}{\partial x}\left(f_{x x} v^{2}\right)
$$

Since we assume that the system is homogeneous in the horizontal direction, we only consider hoppers of narrow width $W$. We also assume $\theta \ll 1$, and the $\theta$ dependences of physical quantities (density, velocity) are calculated up to the first order of $\theta$.

We now study the steady state properties of hoppers. Imposing the steady state condition on the mass conservation Eq. (23) gives

$$
\frac{\partial}{\partial x}(W p v)=0
$$

Also, the momentum conservation Eq. (1b) with the force Eq. (24), in a steady state, becomes

$$
p v \frac{\partial}{\partial x} v=-p g+\frac{1}{W} v^{2}\left(f_{x y} \cos \theta+f_{x x} \sin \theta \cos \theta\right)-\frac{D}{W} \frac{\partial}{\partial x}\left(f_{x x} v^{2}\right) .
$$


We want to know the density and velocity fields which satisfies Eq. (25). We start with a trial solution

$$
\begin{aligned}
& p(x)=p_{o}+A(x) \theta \\
& v(x)=v_{s}+B(x) \theta,
\end{aligned}
$$

where $v_{s}=-\sqrt{p_{o} g W_{o} / f_{x y}\left(p_{o}\right)}$, the steady state velocity for tubes Eq. (12). Substituting Eq. (26) into Eq. (25), and ignore terms of higher order than $\theta$,

$$
\begin{gathered}
v_{s} W_{o} \frac{d A}{d x}+p_{o} W_{o} \frac{d B}{d x}=-2 p_{o} v_{s} \\
D v_{s}^{2} \frac{d f_{x x}}{d p} \frac{d A}{d x}+\left(2 D v_{s} f_{x x}+p_{o} v_{s} W_{o}\right) \frac{d B}{d x}+g W_{o} A-2 v_{s} f_{x y} B=v_{s}^{2} f_{x x}-2 x g p_{o} .
\end{gathered}
$$

Since $f_{x x}$ and $f_{x y}$ are also functions of $p(x)$, one has to know the exact form of these functions to find the solutions of the equation Eq. (27). Here, we assume that $f_{x x}(p)$ can be approximated to $f_{x x}^{o} p^{m}$ for the range of densities found in a hopper. Similarly, $f_{x y} \simeq f_{x y}^{o} p .^{m}$ Since $\theta \ll 1$, the changes of $A(x)$ and $B(x)$ to $x$ will be also slow. We therefore consider variations up to the first order of $x$, that is, $A(x)=A_{o} x+A_{1}$ and $B(x)=B_{o} x+B_{1}$. Substituting $f_{x x}, f_{x y}$ and $A(x), B(x)$ into Eq. (27), we obtain

$$
\begin{aligned}
v_{s} A_{o}+p_{o} B_{o}= & -2 \frac{p_{o} v_{s}}{W_{o}} \\
2 g p_{o}= & \left(m v_{s}^{2} f_{x y}^{o} p_{o}^{m-1}-g W_{o}\right) A_{o}+2 v_{w} f_{x y}^{o} p_{o}^{m} B_{o} \\
v_{s} p_{o} W_{o} B_{o}= & -g W_{o} A_{1}+v_{s}^{2} p_{o}^{m} f_{x x}^{o}+m f_{x y}^{o} v_{s}^{2} p_{o}^{m-1} A_{1} \\
& +2 f_{x y}^{o} v_{s} p_{o}^{m} B_{1}-D f_{x x}^{o} v_{s} p_{o}^{m}\left(2 B_{o}+\frac{m v_{s} A_{o}}{p_{o}}\right) .
\end{aligned}
$$

There are only three conditions in Eq. (28) for four unknown variables. The other condition comes from the freedom in choosing the origin of the $x$ axis. We set the origin so that $B_{1}=0$. Then, the solution of Eq. (28) is

$$
\begin{aligned}
& B_{o}=\frac{2 m}{3-m} \cdot \frac{v_{s}}{W_{o}} \\
& A_{o}=-\frac{p_{o}}{W_{o}} \cdot \frac{6}{3-m} \\
& A_{1}=\frac{v_{s}^{2}}{(m-2) g W_{o}} \cdot\left[\frac{2 m}{3-m}\left(p_{o}-f_{x x}^{o} p_{o}^{m} \frac{D}{W_{o}}\right)-f_{x x}^{o} p_{o}^{m}\right] .
\end{aligned}
$$


Intuitive solutions for hoppers are that the magnitude of the velocity increases, and the density decreases as $x$ is increased. Although the solution Eq. (29) becomes intuitive one for $0<m<3$, it can also have very different behaviors for other ranges of $m$.

Having obtained the steady state for hoppers, we now proceed to study kinetic waves. The density and velocity fields are described by Eq. (26) with one free parameter $p_{o}$. Consider two regions of density, where $p_{o}$ is chosen to be $p_{1}$ and $p_{2}$, respectively. The velocity of the interface $U_{h}$ between the regions, which can be calculated following the way described in Sec. IIA, is

$$
U_{h}=\frac{\Delta j}{\Delta \rho}=\frac{\Delta\left[p_{i} v_{s}\left(p_{i}\right)+v_{s} p_{i} A_{1}\left(p_{i}\right) \theta+\left(p_{i} B_{o}\left(p_{i}\right)+v_{s}\left(p_{i}\right) A_{o}\left(p_{i}\right)\right) x \theta\right]}{\Delta\left[p_{i} v_{s}\left(p_{i}\right)+\left(A_{o}\left(p_{i}\right)+A_{1}\left(p_{i}\right) x\right) \theta\right]} .
$$

We consider small fluctuations of density: $p_{1}=p$ and $\delta p=p_{2}-p \ll 1$. The velocity, up to the first order of $\theta$, is

$$
\begin{aligned}
U_{h} \simeq \frac{d j}{d \rho} & =\frac{d}{d p}\left(p v_{s}(p)\right)+\frac{d}{d p}\left(A_{1}(p) v_{s}(p)\right) \theta \\
& +\frac{d}{d p}\left(p B_{o}(p)+v_{s}(p) A_{o}(p)\right) x \theta-\frac{d}{d p}\left(p v_{s}(p)\right) \frac{d}{d p}\left(A_{o}(p) x+A_{1}(p)\right) \theta
\end{aligned}
$$

The above velocity $U_{h}$ is calculated at position $x$. One should note that the density at the position is not $p$ but $p+\left(A_{o}(p) x+A_{1}(p)\right) \theta$. We now compare the velocity of density waves in hoppers and tubes. The velocity of density waves in tubes $U_{t}$ at the above density is

$$
U_{t}=\frac{d}{d p}\left(p v_{s}(p)\right)+\left(\frac{d v_{s}(p)}{d p}+p \frac{d v_{s}(p)}{d p}+p \frac{d^{2} v_{s}(p)}{d p^{2}}\right)\left(A_{o}(p) x+A_{1}(p)\right) \theta,
$$

which is give by Eq. (13).

Therefore, density waves also exist in hoppers, and their velocity is given by $U_{h}(\theta, p, x)=$ $U_{t}+C(p, x) \theta$, where $C(p, x)$ is a complicated function of $x$ and $p$.

\section{MOLECULAR DYNAMICS SIMULATIONS}

\section{A. Interactions between particles}

We discuss the interaction between the particles used in the MD simulations of granular flows. The force between two particles $i$ and $j$, in contact with each other, is the following. 
Let the coordinate of the center of particle $i(j)$ to be $\vec{R}_{i}\left(\vec{R}_{j}\right)$, and $\vec{r}=\vec{R}_{i}-\vec{R}_{j}$. In two dimensions, we use a new coordinate system defined by the two vectors $\hat{n}$ (normal) and $\hat{s}$ (shear). Here, $\hat{n}=\vec{r} /|\vec{r}|$, and $\hat{s}$ is defined as rotating $\hat{n}$ clockwise by $\pi / 2$. The normal component $F_{j \rightarrow i}^{n}$ of the force acting on particle $i$ by $j$ is

$$
F_{j \rightarrow i}^{n}=k_{n}\left(a_{i}+a_{j}-|\vec{r}|\right)^{3 / 2}-\gamma_{n} m_{e}(\vec{v} \cdot \vec{n})
$$

where $a_{i}\left(a_{j}\right)$ is the radius of particle $i(j), m_{i}\left(m_{j}\right)$ the mass of particle $i(j)$, and $\vec{v}=d \vec{r} / d t$. The first term is the Hertzian elastic force, where $k_{n}$ is the elastic constant of the material. And, the constant $\gamma_{n}$ of the second term is the friction coefficient of a velocity dependent damping term, $m_{e}$ is the effective mass, $m_{i} m_{j} /\left(m_{i}+m_{j}\right)$. The shear component $F_{j \rightarrow i}^{s}$ is given by

$$
F_{j \rightarrow i}^{s}=-\gamma_{s} m_{e}(\vec{v} \cdot \vec{s})-\operatorname{sign}(\delta s) \min \left(k_{s}|\delta s|, \mu\left|F_{j \rightarrow i}^{n}\right|\right),
$$

where the first term is a velocity dependent damping term similar to that of Eq. (33a). The second term is to simulate static friction, which requires a finite amount of force $\left(\mu F_{j \rightarrow i}^{n}\right)$ to break a contact 34]. Here, $\mu$ is the friction coefficient, $\delta s$ the total shear displacement during a contact, and $k_{s}$ the elastic constant of a virtual spring. There are several studies on granular systems using similar interactions. However, only a few of them [34 36] include static friction. A particle can also interact with a wall. The force on particle $i$, in contact with a wall, is given by Eqs. (33) with $a_{j}=\infty$ and $m_{e}=m_{i}$. A wall is assumed to be rigid, i.e. it is not affected by the collisions with particles. Also, the system is under a gravitational field $\vec{g}$. We do not include the rotation of the particles in present simulation. A detailed explanation of the interaction is given elsewhere [36].

\section{B. Density waves in vertical tubes}

\section{Waves without static friction}

We first simulate granular flows without static friction. Thus, we set $\mu=0$, and the shear force is only due to the velocity dependent friction term in Eq. (33b). We study the 
system in two dimensions. Tubes are modeled by two parallel sidewalls of length $L$, and separated by distance $W$, between which particles flow (Fig. ॠ). We use a periodic boundary condition in the vertical direction. Particles come out of the bottom of the tube are fed into the top. In order to avoid a haxagonal packing formed by monodisperse particles, we use polydisperse particles, whose radii are drawn from the gaussian distribution of mean 0.1 and width 0.02 . We initially arrange particles to be equally spaced along the vertical direction, and calculate the positions and the velocities of the particles at subsequent steps using a fifth-order predictor-corrector method.

In Fig. 5, we show the time evolutions of the densities and the velocities of the particles with $W=1, L=15$ and the number of particles $N=225$. The density plots are made as follows. We divide the tube into several regions (bins) of equal height (typically, 5 times particle diameter), and count the number of particles $n_{i}$ in bin $i$. We set the grayscale of each bin to be proportional to $n_{i}$. We choose white for $n_{i}=d_{l}$, and black for $n_{i}=d_{u}$, where $d_{l}\left(d_{u}\right)$ is the lower (upper) bound for $n_{i}$. If $n_{i}$ is smaller than $d_{l}$ or larger than $d_{u}$, the grayscale is chosen to be white or black, respectively. In Fig. 5(a), we use $d_{l}=0$ and $d_{u}=30$. The density field at a given time step is plotted as one horizontal line, where boxes of different grayscale represents bins of the tube. Here, the leftmost box corresponds to the bin at the bottom of the tube. The velocity plot is made using the same procedure as above, except the grayscale is proportional to the average vertical velocity $v_{i}$ in bin $i$. In order to enhance the contrast, we subtract the center of mass velocity from $v_{i}$. We choose white for $v_{l}$ and black for $-v_{l}$. Here, we set $v_{l}=60$. The time step is chosen to be $5.0 \times 10 .^{-5}$ The time interval between the successive raws in the density and velocity plots is 100 iterations. The parameters for the simulation are $k_{n}=1.0 \times 10^{6}, k_{s}=1.0 \times 10^{4}$ and $\gamma_{n}=\gamma_{s}=500$ between the particles. Between the particles and the walls, we use $k_{n}=5.0 \times 10^{6}$ in order not to allow the particles go through the walls, while the other parameters are kept to be the same.

In Fig. 5, one can see a region of high density is being formed from the homogeneous system [37]. Also, a high density region may be split into two, or two regions may merge to 
form one region. However, for the most of time, these density fluctuations just travel with almost constant velocity. These traveling density patterns are first observed in simulations by Pöschel [26]. Comparing Fig. 5(a) and (b), one can notice correlations between the density $n_{i}$ and the velocity $v_{i}$. The particles seem to travel slower (faster) in high (low) density regions, which is very similar to traffic flows. This correlation is one hint that the density waves may be of kinetic nature. In order to systematically study the correlation, we measure the density dependence of the flux in a steady state. We choose a bin, and we calculate the friction and gravity force acting on the bin. Since we want to measure the flux in a steady state (the total force is zero), we require the total force (sum of the friction and the gravity) is smaller than $r$ times the gravity, and we discard bins if the requirement is not fulfilled. For bin $i$ in a steady state, we measure the total flux, defined as $j_{i}=v_{i} n_{i}$. We calculate the density $n_{i}$ dependence of the average flux $j_{i}$ for a system of fixed total number of particles $N$. Here, the averages are taken over time. We measure the flux-density curve for several different values of $r$ ranging from 0.1 to $\infty$. The results are not very sensitive to the values of $r$, when we study the systems in a steady state. We set $r=1.0$ from now on. We also measure the curve for several values of $N=150,225,280,337$. For large $N$, we have accurate estimate of $j_{i}$ for large values of $n_{i}$, but poor one for small $n_{i}$. The situation is opposite for the systems of small $N$. For the intermediate values of $n_{i}$, however, all the systems give good estimates, which agree with each other. In Fig. 6, we show the flux-density curve averaged over the four values of $N$. The fact that we have a well defined flux-density curve suggest the system is in a steady state, which implies that kinetic wave is sufficient for the description of the evolutions of the system. Furthermore, the curve resembles that of a traffic flow, which is considered to be one of the typical examples of kinetic waves [30.

One additional evidence that the density waves are of kinetic nature is the dependence of the velocity of the waves on the average density. The velocities of kinetic waves are given by Eq. (8), which are the slope of the flux-density curve, for small density fluctuations. From Fig. 6, we expect that the velocity is a large negative value for a small density, approaches to zero, and becomes a large positive number as the density is increased. We directly measure 
the velocities of the waves from the slopes of high density regions like the one shown in Fig. 5 . In Table. I, we show the average velocities for several values of $N$, where the average density $<n_{i}>$ is given by $N / L$. In the table, one note the velocity is negative $(-41)$ for small $N$, and is increased to positive (113) for large $N$, which is exactly the way predicted by the theory of kinetic wave. Furthermore, the measured velocities are consistent with the local slopes of the flux-density curve Fig. 6, although the slopes can not be accurately determined due to the large error bars. Based on the above evidences, as well as the theoretical argument given in Sec. [IB 1, we conclude the above density waves found in vertical tubes are kinetic waves.

We also want to discuss the origin of fluctuations in the system. As shown above, density fluctuations (waves) are formed from an uniform density system. But kinetic waves, as discussed in Sec. [IA, can not create fluctuations. Also, some of the waves are split into two waves, which can not be described by the evolutions of kinetic waves alone. There must be some sources of fluctuations or "noises" in the system. Since the system is deterministic, one might think the system can not have noises. The "noises" come from the fact that the equations of motion Eq. (II) as well as the form of the friction force are relations between averaged quantities. The fluctuations around their averaged values, especially relevant in small scale descriptions of systems, are identified as "noises".

\section{Waves with static friction}

We next study the flows through vertical tubes with static friction. As discussed in Sec. [IB 1, we expect two types of behaviors depending on the friction coefficient $\mu$. If $\mu>\rho g W / P_{s}$, the system can not move, and stays as a static column. Otherwise, the particles start move, and forms periodic stagnant zones whose separation $\mathcal{D}(p)$ is determined by Eq. 18. The setup we used in the MD simulations is exactly the same as the previous one (Fig. (1), where static friction is introduced by choosing a non-zero $\mu$. Between the particles, we use $\mu_{P P}=0.5$. We also set the shear friction coefficient $\gamma_{s}$ to be zero. We do simulations 
with $W=1$ and $L=15$, starting with $N=225$ particles. All the other parameters are kept to be the same as above. In the simulations, we find three types of behaviors depending on the friction coefficient between the walls and the particles $\mu_{W P}$ : (1) If $\mu_{W P} \geq 1.0$, the particles can move initially, but they eventually form static column(s). (2) On the other hand, if $\mu_{W P} \leq 0.6$, the particles constantly increase their speeds, and do not go into a steady state until the end of simulations (50 000 iterations). (3) In the intermediate regime, $0.6 \leq \mu_{W P} \leq 1.0$, we find steady states, where density waves travels with almost constant velocities. The static structures found in the first regime are precisely what is expected from the theory. In the second regime, however, the systems do not reach steady states, in contrast to the theory. One possibility is that the time needed to reach a steady state is larger than the simulation time. To check this possibility we do longer simulations of the system with $\mu_{W P}=0.6$, which does not reach a steady state in 50000 iterations. We find the system does reach a steady state at around 100000 iterations.

In the third regime, the simulations with $L=15$ do not show the expected periodic stagnant zones but traveling density fluctuations. For example, we show the density evolutions in a tube of $L=15$ in Fig. [7(a), where $\mu_{W P}=0.9$ and the time interval between the successive raws are 0.0025. A region of high density travels with almost constant velocity of $-86 \pm 13$. There are few possibilities to understand the discrepancy. First of all, there can be a problem of commensurability. In general, the length of the tube $L$ is not an integer multiple of the stagnant zone separation $\mathcal{D}(p)$. Thus, the distance between the clogged zone can not all be $\mathcal{D}(p)$ due to the periodic boundary condition. It can be shown, following the argument in Sec. [IB 1, that the distances between the stagnant zones are all $\mathcal{D}(p)$ except one, which is smaller than $\mathcal{D}(p)$. This configuration, however, can not be a steady state. The stagnant zone just below the small separation becomes unstable, since the incoming velocity of the particles are smaller than $v_{c}(p)$. These particles travel further down to reach $v_{c}(p)$, then form a stagnant zone. In effect, the stagnant zones travels down, if $L$ is not an integer multiple of $\mathcal{D}(p)$. There also can be problems of "noises." As discussed before, there are noises to the continuum description we have been using. Due to these noises, the 
separations between the zone are not all equal, but fluctuate around $\mathcal{D}(p)$. Furthermore, the noise also cause the stagnant zones to move around. Considering the above possibilities, we expect that several stagnant zones in a long tube drift downwards, whose separations fluctuate around $\mathcal{D}(p)$. In order to check the possibilities, we study density waves for several values of $L$, keeping the average density constant. The evolution of density field for $L=30$ is shown in Fig. 7(b). One can see a region of high density is traveling downwards with the velocity around -25 . The results for both $L=15$ and $L=30$ are consistent with the prediction. Since there are only one region of high density, we expect $\mathcal{D}(p)$ is larger than $L$. We then expect the velocities of the density waves are always negative, and their magnitude decreases as one increases $L$, thereby approaching $\mathcal{D}(p)$. The situation for $L=45$ is quite different, as shown in Fig. 7(c). The high density region seems to travel upwards, and the velocity seems to fluctuate more. These two features are also found in a few additional runs we study for $L=45$. The fluctuations can be caused by the "noise," but the trend of moving upwards can not be explained. We also studied the system with $\mu_{W P}=0.7$ and 0.8 , and find essentially the same.

\section{Comparison with the experiments}

We now discuss the results of the experiments with vertical tubes, and compare them with the theoretical and simulational results obtained above. We first discuss the experiments. Density waves in vertical tubes are first found in the experiments by Pöschel [26]. He found two types of waves: (1) Regions of large densities occur at random positions, and they travel with non-zero velocity. (2) The separations between the regions of large densities seem to be about the same, and they fluctuate around certain positions 38. The conditions needed to obtain each types of waves are unknown. The flows are also studied in vacuum, and surprisingly, the density waves disappear [39,40]. It is still not understood how air affects the formation of density waves. Here, we discuss one of the possible mechanisms.

The conditions for forming kinetic waves, as discussed in Sec. ПВ 1, are fairly simple. One 
important condition is that the friction force should balance the driving force, which in this case, gravity. It is possible that the friction force by the sidewalls is too small to balance gravity. Consider a block of particles falling down in a tube. If the density of the block is large enough, air can not easily pass through the block, and the air pressure just behind the block can be smaller than that of the front. The pressure difference gives rise a force to slow down the block, which acts as an additional friction force. Since the pressure difference is expect to increase by increasing the density and increasing the velocity of the block, the friction force by air can balance gravity at high velocity, and the balance produces kinetic waves. As one can see, this argument is largely speculative, and should be checked by careful experiments. Especially, it should be check whether there is pressure difference between the front and the behind of a moving granular blocks of high density, and whether the difference is enough to balance the gravity.

Assuming the above mechanism to be hold, how can one understand the two types of waves found in the experiments? The two waves are readily compared with the predicted kinetic waves with and without static friction. If $\mathcal{D}(p)$ is larger than the length of the tube, we do not expect to see any stagnant zone (for open boundary condition). Therefore, we effectively see the system without static friction, which produces traveling kinetic waves. However, if $\mathcal{D}(p)$ is much smaller than the tube length, we do see periodic stagnant zones. The distance $\mathcal{D}(p)$ is a decreasing function of the friction (both by the sidewalls and air), and it is possible that the tube which produces the periodic zones have larger friction than others. This possibility again is speculative, and should also be checked by more controlled experiments. For example, the friction can be changed by using the sidewalls of varying surface roughness. If air is responsible for an additional friction, one can also change the friction by controlling the pressure of air. 


\section{Dynamic waves}

We conclude this section by discussing dynamic waves in granular flows through tubes. As discussed in Sec. IIB2, we expect the granular flows become less stable under density fluctuations for smaller coefficient of restitution $e$. Since $e$ is small for large normal damping coefficient $\gamma_{n}$, we expect the system to be less stable for large $\gamma_{n}$. We simulate the system with several values of $\gamma_{n}=5 \times 10^{2}, 1 \times 10^{3}, 2 \times 10^{3}, 3 \times 10^{3}$, and study the stability. In Fig. 8, we show the density fields of tube with $L=15$ and $N=225$ for several values of $\gamma_{n}$. Here, the time intervals between the successive raws are 0.0025. We do not find any sign of dynamic waves, even for longer simulations. We also repeat the simulations with higher density $N=337$, and find that the results are essentially the same. The coefficient of restitution $e$ depends on the relative velocity between the colliding particles for the Hertzian contact force. Considering the scale of velocities found in tubes, $e$ is roughly estimated to be 0.1 for $\gamma_{n}=3 \times 10 .{ }^{3}$ It is possible that the dynamic instability occur at even larger values of $\gamma_{n}$. The limitation of the constant timestep type algorithm we are using is that we have to decrease the timestep to prevent a numerical instability, if we want to increase $\gamma_{n}$. For

example, the timestep is chosen to be $5 \times 10^{-5}$ for $\gamma_{n}=5 \times 10^{2}$, and $1 \times 10^{-5}$ for $\gamma_{n}=3 \times 10^{3}$. Therefore, we can not simulate systems of arbitrarily large $\gamma_{n}$. One have to use event driven type algorithms (for example, Ref. [24]) to overcome this limitation.

\section{Density waves in hoppers}

In Sec. 【IC, we presented a theory which predicts the existence of kinetic waves in granular flows through hoppers. In that section, the velocity of the kinetic wave in hoppers of opening angle $2 \theta$ is shown, up to the first order of $\theta$, to be $U_{h}(\theta, p, x)=U_{t}(p)+C(p, x) \theta$. Here, $p$ is the packing fraction and $x$ the position, and $U_{t}(p)$ the velocity of kinetic waves in tubes, and $C(p, x)$ is a complicated function of $p$ and $x$. We thus expect $U_{h}$ for a hopper of small $\theta$ is not very different from that of tubes. The conditions for the existence of dynamic 
waves are too difficult to be obtained from the theory.

We study hoppers of length $L=15$, bottom width $W_{o}=1.0$, and several values of the opening angle $2 \theta$. We apply a periodic boundary condition in the vertical direction. The particles come out of the bottom are again fed into the top. The boundary condition, which is not natural for the hopper geometry, can introduce some artifacts to the system. We later check the results by comparing with those from the open boundary condition. The main reason for using the periodic boundary condition is that we can simulate the systems for longer time, which in effect study the systems of larger sizes without actually increasing the length $L$ (and the number of particles). We initially arrange particles as a square lattice in a hopper. The lattice constant is 0.1 , the average radius of the particles. The average number of particles per unit area is very close to 25 , the maximum density allowed for the square packing. The randomness in the initial configuration is introduced by the polydispersity of the particles. The interaction parameters are chosen to be exactly the same as those for tubes.

We first study the system without static friction. The static friction coefficient $\mu$ is chosen to be 0 , and the velocity dependent shear friction term $\gamma_{s}$ to be 500. In Fig. 9, we show the time evolutions of the density and the velocity fields in hoppers. The plots are made in the same way as Fig. 5 except the fact that the density is obtained by dividing the number of particles in a bin by the area of the bin, which is not constant for a hopper. The grayscales are chosen to show clear contrast between the low and high density (velocity) regions by controlling $d_{l}$ and $d_{u}\left(v_{l}\right)$.

In Fig. 9(a) and (b), we show the density and the velocity fields for a tube $(\theta=0)$ in order to serve as a reference to compare with those for hoppers. The time interval between the successive lines are 0.005. In Fig. 9(c) and (d), the fields for a small angle hopper $\left(\theta=1^{\circ}\right)$ are shown. In the density plot (Fig. 9(c)), there are two waves in the hopper, one travels upwards and the other downwards. Comparing with the velocity plot (Fig. 9(d)), the densities of the upward wave are strongly correlated to the velocities, while there seems to be no such correlation for the downward waves. The correlations found in the upward waves 
are qualitatively the same as that of kinetic wave, i.e., the particles travel slow (fast) in the regions of high (low) density. It is quite possible that the upward wave is of kinetic nature. Also, the velocities of kinetic waves in hoppers with small opening angle are expected to be very close to that of tubes. The fact that the velocity of the upward waves $(113 \pm 4)$ are very close to that of the tube $(93 \pm 10)$ is one other support that the wave is kinetic [41. We now consider the waves travel downwards. The waves, not only show no correlations between the density and the velocity fields, but also travels in the opposite direction to the kinetic waves in the tube (Fig. 9(a)), which suggests that the waves are not probably kinetic. Furthermore, the density contrasts of the waves are constantly increasing, which is not possible for kinetic waves. The increments can be more clearly seen in hoppers of larger opening angle as shown in (e)-(f) $\left(\theta=4^{\circ}\right)$. The downward waves initially coexist with the kinetic waves, but eventually dominate the system. The above facts are still true for the largest $\theta=10^{\circ}$ we study. The properties of the downward waves listed above suggest that the downward waves are of dynamic nature. According to the argument used for the dynamic waves in tubes, dynamic waves will be more easily formed using non-elastic particles. We check this possibility by studying a hopper of $\theta=1^{\circ}$ for different values of $\gamma_{n}$. In Fig. 10, we show the density fields for $\gamma_{n}=1 \times 10^{3}$ and $2 \times 10^{3}$. Comparing with that of $\gamma=5 \times 10^{2}$ (Fig. 9) (c), one can see the waves for larger $\gamma_{n}$ are indeed formed earlier, and their intensities (density contrast) are larger. This add one more support that the downward waves are dynamic. We also measure the velocities of the dynamic waves for several values of $\theta$. As shown in Table [II, the magnitude of the velocity is decreased as $\theta$ is increased. Following the trend, it is quite possible that the velocity becomes zero for finite $\theta$, and change its sign.

We now study hoppers with the open boundary condition. In Fig. 11(a) and (b), we show the density and the velocity fields for a hopper of $\theta=1^{\circ}$ and $L=30$. Here, we turn off static friction $(\mu=0)$, and we set $\gamma_{s}=5 \times 10^{2}$. One can hardly see any fluctuations in the density field, but one can see some traveling patterns in the velocity field. We now turn on static friction. We set $\gamma_{s}=0$ and $\mu \neq 0$. The density and the velocity fields with $\mu=0.5$ are shown in Fig. 11(c) and (d). One can now see upward traveling density waves. Furthermore, the 
density field have strong correlations with the velocity field, suggesting that the waves are kinetic. Even though kinetic waves are present in the system independent of static friction, the amplitudes of the waves are too small to be visible without it. Static friction provides an effective mechanism for creating large density fluctuations. Also, the density waves are not present in the hoppers of small $L$. The absence of density waves can be caused by the fact that the particles need to travel certain distances to reach a steady state around which the density waves can only be seen. All these results remain valid for larger values of $\theta$ we have studied. For example, we show the case of $\theta=5^{\circ}$ in Fig. 11(e)-(f).

We also search for the dynamic waves in hoppers with the open boundary condition. We simulate the system with several values of $\gamma_{n}$, and check the instability of creating dynamic waves. For the range of $\gamma_{n}$ we have studied $\left(5 \times 10^{2} \sim 3 \times 10^{3}\right)$, we do not find the instability. Again, the simulations for larger values of $\gamma_{s}$ are limited due to the algorithm we are using. Even though we do not rule out dynamic waves in hoppers in general, the dynamic instability found above seems to be an artifact of the periodic boundary condition.

We now compare the results with the experiments. Baxter et al found density waves in a hopper only if a certain amount of rough sands is mixed with smooth sands [28]. The role of the rough sands is not clearly established. It is possible that the rough sands forms local "arches", thereby increasing density fluctuations. In that case, the role played by the rough sands is the same as that of static friction, which provides density fluctuations to maintain kinetic waves. Also this is consistent with the fact that the amplitudes of the density waves are a smooth function of the fraction of the rough sands, which suggests that the density waves in the experiments are kinetic. The suggestion, of course, has to be checked by experiments, for example, by measuring the correlations between the density and the velocity fields. 


\section{DISCUSSION}

We have presented theoretical and numerical evidences that the density waves found in the simulations of Pöschel is of kinetic nature. However, the density waves found in the experiments are not fully understood. The first and foremost problem is to find the form of the friction force. In the MD simulations, the friction force is generated by the collisions of particles to the sidewalls. In experiments, the collisional friction force seems to be too small compared to gravity, and another friction force related to air gives dominant contribution. We proposed a mechanism how air can generates a friction force, which should be checked by experiments. We want to emphasize that since the existence of kinetic wave is not strongly dependent on the details of the friction force, the density waves found in the experiments are very likely to be kinetic waves.

We find conditions for the existence of dynamic waves in tube. The conditions depend on details of the force, which we do not know. We numerically search for dynamic waves by increasing $\gamma_{n}$, since we expect dynamic waves can be more easily created for inelastic particles. Even for the largest $\gamma_{n}$ we studied, we can not find the dynamic waves. It is possible that dynamic waves occur for even larger values of $\gamma_{n}$. We are not able to check the possibility, since the algorithm we are currently using becomes very inefficient for larger values of $\gamma_{n}$.

We also have present theoretical and numerical evidences that there are kinetic waves in hoppers. Especially, the kinetic waves in hoppers with the open boundary condition are visible only with static friction. This can be readily compared with the fact that one need finite fraction of rough sands to observe the density waves in the experiments. Here, the role of rough sands can be creating large density fluctuations to maintain kinetic waves just like static friction. The suggestion that the density waves in the experiments of hoppers are kinetic waves should be checked, for example, by studying the correlation between the density and the velocity fields.

We are not able to do a linear stability analysis for hoppers, due to the complicated 
density and velocity fields in the steady state. In the simulations with the peridic boundary condition, we find another density waves, which we believe to be dynamic on a few evidences. Since we do not find the dynamic waves with the open boundary conditions, we think the above dynamic wave is an artifact of the boundary condition. However, we do not rule out the dynamic waves in hoppers, especially for large values of $\gamma_{n}$.

I thank Michael Leibig, Hans Herrmann and Thorsten Pöschel for many useful discussions. 


\section{REFERENCES}

[1] S. B. Savage, Adv. Appl. Mech. 24, 289 (1984); S. B. Savage, Disorder and Granular Media ed. D. Bideau, North-Holland, Amsterdam (1992).

[2] C. S. Campbell, Annu. Rev. Fluid Mech. 22, 57 (1990).

[3] H. M. Jaeger and S. R. Nagel, Science 255, 1523 (1992).

[4] P. Evesque and J. Rajchenbach, Phys. Rev. Lett. 62, 44 (1989).

[5] C. Laroche, S. Douady and S. Fauve, J. Phys. (France) 50, 699 (1989).

[6] E. Clement, J. Duran and J. Rajchenbach, Phys. Rev. Lett. 69, 1189 (1992).

[7] J. Lee, HLRZ preprint 101/92 (1992).

[8] G. Rátkai, Powder Technol. 15, 187 (1976).

[9] S. B. Savage, J. Fluid Mech. 194, 457 (1988).

[10] O. Zik and J. Stavans, Europhys. Lett. 16, 255 (1991)

[11] Y-h. Taguchi, Phys. Rev. Lett. 69, 1371 (1992).

[12] J. A. C. Gallas, H. J. Herrmann and S. Sokołowski, Phys. Rev. Lett. 69, 1375 (1992).

[13] J. C. Williams, Powder Technol. 15, 245 (1976).

[14] P. K. Haff and B. T. Werner, Powder Technol. 48, 239 (1986).

[15] A. Rosato, K. J. Strandburg, F. Prinz and R. H. Swendsen, Phys. Rev. Lett. 49, 59 (1987).

[16] P. Devillard, J. Phys. (France) 51, 369 (1990).

[17] R. Jullien and P. Meakin, Phys. Rev. Lett. 69, 640 (1992).

[18] J. Duran, J. Rajchenbach and E. Clémont, Phy. Rev. Lett. 70, 2431 (1993). 
[19] R. A. Bagnold, Proc. R. Soc. London A 225, 49 (1954).

[20] S. Savage and M. Sayed, J. Fluid Mech. 142, 391 (1984).

[21] C. Campbell, J. Fluid Mech. 203, 449 (1989).

[22] M. Babić, H. Shen and H. Shen, J. Fluid Mech. 219, 81 (1990).

[23] P. A. Thompson and G. S. Grest, Phys. Rev. Lett. 67, 1751 (1991).

[24] M. A. Hopkins, M. Y. Louge, Phys. Fluids A 3, 47 (1990).

[25] S. Savage, J. Fluid Mech. 241, 109 (1992).

[26] T. Pöschel, HLRZ preprint 89/92 (1992).

[27] J. O. Cutress and R. F. Pulfer, Powder Technol. 1, 213 (1967).

[28] G. W. Baxter and R. P. Behringer, T. Fagaert and G. A. Johnson, Phys. Rev. Lett. 62, 2825 (1989).

[29] G. Ristow, J. Physique I 2, 649 (1992); G. Ristow and H. J. Herrmann, HLRZ preprint $7 / 93(1993)$.

[30] M. J. Lighthill and G. B. Whitham, Proc. Roy. Soc. A 229, 281 and 317 (1955).

[31] W. H. Press, B. P. Flannery, S. A. Teukolsky, W. T. Vetterling, Numerical Recipes (Cambridge, 1986).

[32] Similar stagnant zone is also found in shear cell simulations of Y. Zhang, C. S. Campbell [J. Fluid Mech. 237, 541 (1992)].

[33] I. Goldhirsch and G. Zanetti, Phys. Rev. Lett. 70, 1619 (1993).

[34] P. A. Cundall and O. D. L. Strack, Géotechnique 29, 47 (1979).

[35] Y. M. Bashir and J. D. Goddard, J. Rheol. 35, 849 (1991).

[36] J. Lee and H. J. Herrmann, J. Phys. A 26, 373 (1993). 
[37] The formation of large density regions from random fluctuations in the density field can be explained by the theory of kinetic waves [M. Leibig, HLRZ preprint 42/93].

[38] T. Pöschel, private communication.

[39] D. Bideau, private communication.

[40] A. Hansen, private communication.

[41] The vertical size of pixels in Fig. 9(a) and (b) is twice as large as compared with Fig. 9(c) - (f). The velocity of upward waves in Fig. 9(a) is comparable to that of Fig. 9(c). 


\section{FIGURES}

FIG. 1. The time evolution of the interface between the uniform-flows with density $\rho_{a}$ and $\rho_{b}$.

FIG. 2. Time evolution of density fluctuation with the force given by Eq. (2): (a) Dynamic waves are decaying, while the kinetic waves survives $(\mu=2, \nu=-1)$, (b) Dynamic waves are growing, and take over kinetic waves $(\mu=-2, \nu=-1)$.

FIG. 3. (a) Hopper with the opening angle 20. (b) The friction force per unit length on the wall is sum of $\tau_{x y} \cos \theta$ (left) and $\tau_{x x} \cos \theta \sin \theta$ (right).

FIG. 4. Vertical tubes in two dimension with width $W$ and length $L$ under gravity. We apply a periodic boundary condition in the vertical direction.

FIG. 5. Time evolutions of (a) density and (b) velocity fields of particles in a tube of $W=1, H=15$. Fields at a given time is shown as a horizontal line of boxes. The grayscale of each box is proportional to the density and the velocity in that region of the tube. Regions of high density are formed, and travel with almost constant velocity.

FIG. 6. The flux-density curve for a tube of $W=1, H=15$ averaged over time and different values of $N$. The parabolic shaped curve resembles that found in a traffic jam.

FIG. 7. The evolution of density fields in tubes with $\mu=0.5$ and (a) $L=15$, (b) 30 and (c) 45 . High density regions travel downwards in (a) and (b), but travel upwards with larger fluctuations in (c).

FIG. 8. Density fields for tube with $L=15$ and $N=225$ for several values of $\gamma_{n}$ : (a) $\gamma_{n}=1 \times 10^{3}$, (b) $2 \times 10^{3}$ and (c) $3 \times 10^{3}$. There is no sign of dynamic instability.

FIG. 9. The density and velocity fields for hoppers with different opening angle. (a)-(b) $\theta=0^{\circ}$, (c)-(d) $1^{\circ}$, (e)-(f) $4^{\circ}$. For small $\theta$, there are two waves traveling in opposite directions, where the downward waves eventually dominate. 
FIG. 10. The density fields for a hopper of $\theta=1^{\circ}$ with (a) $\gamma_{n}=1 \times 10^{3}$ and (b) $2 \times 10^{3}$. The intensities (density contrast) of waves are larger for larger $\gamma_{n}$.

FIG. 11. The density and velocity fields for a hopper with the open boundary condition. In (a)-(b) the fields for a hopper of $\theta=1^{\circ}$ without static frcition, (c)-(d) for $\theta=1^{\circ}$ with static friction, and (e)-(f) for $\theta=5^{\circ}$ with static friction are shown. 


\section{TABLES}

TABLE I. The velocity of kinetic waves in a tube for several values of the average density

\begin{tabular}{lcc}
\hline \hline $\mathrm{N}$ & $\left\langle n_{i}\right\rangle$ & Velocity of kinetic wave \\
\hline 150 & 10.0 & $-41.0 \pm 2.0$ \\
225 & 15.0 & $5.0 \pm 9.0$ \\
280 & 18.7 & $12.0 \pm 11.0$ \\
337 & 22.5 & $113.0 \pm 4.0$ \\
\hline \hline
\end{tabular}

TABLE II. The velocity of dynamic waves in a hopper for several values of $\theta$.

\begin{tabular}{cc}
\hline \hline$\theta$ (in degrees) & Velocity of dynamic wave \\
\hline 1.0 & $-49 \pm 3$ \\
2.0 & $-40 \pm 5$ \\
4.0 & $-26 \pm 4$ \\
6.0 & $-21 \pm 5$ \\
8.0 & $-18 \pm 4$ \\
\hline \hline
\end{tabular}

